\title{
Extended Temporal Gradient for the Retrograde and Anterograde Amnesia Produced by Ibotenate Entorhinal Cortex Lesions in Mice
}

\author{
Yoon H. Cho, Daniel Beracochea, and Robert Jaffard \\ Laboratoire de Neurosciences Comportementales et Cognitives, CNRS URA 339, Université de Bordeaux I, 33405 \\ Talence Cédéx, France
}

\begin{abstract}
Effects of ibotenic entorhinal cortex (EC) lesions on both retrograde and anterograde amnesia in mice were assessed using two-choice discrimination tasks learned at different intervals before surgery in two eight-arm radial mazes. The results indicated that EC-lesioned mice were severely impaired in postoperative retention of discrimination problems learned $3 \mathrm{~d}$ or 2 weeks prior to surgery, but showed no deficit on problems learned between 4, and up to 6 weeks before surgery, as compared to sham-operated controls. When trained on a novel two-choice discrimination problem (not acquired preoperatively), experimental subjects demonstrated quite normal rates of acquisition, but were impaired in learning its reversal. Furthermore, they exhibited a faster rate of forgetting (anterograde amnesia) relative to controls over a 2-week retention interval. These results indicate that approximately 4 weeks is required before memory for a twochoice spatial discrimination problem no longer depends on the integrity of the entorhinal cortex, and suggests that, beyond this time, an EC-independent memory storage system is capable of supporting the retrieval of information. The data, together with complementary behavioral results, are discussed in the context of current theories of memory storage.
\end{abstract}

IKey words: retrograde amnesia, entorhinal cortex, ibotenate lesions, spatial reference memory, radial maze, mice]

The human amnesic syndrome is characterized by a severe loss of memory for events that occur subsequent to brain insult (anterograde amnesia), together with a much more variable memory impairment for premorbid events (retrograde amnesia).

Neuropsychological studies of both anterograde and retrograde amnesia have been useful in addressing the memory consolidation issue. Thus, it has been suggested that, at least for bitemporal lesions or dysfunction, anterograde amnesia could be attributed to a consolidation deficit, that is, a failure to transform short-term into long-term memory traces. More importantly, these studies have shown that retrograde amnesia could

\footnotetext{
Received Feb. 19, 1992; revised Aug. 27, 1992; accepted Oct. 29, 1992.

This work was supported by the Centre National de la Recherche Scientifique and the Fondation pour la Recherche Médicale. We wish to thank Professor $\mathrm{L}$. E. Jarrard for his advice and evaluation of ibotenic acid entorhinal cortex lesions. A part of these results were presented at the 21 st Annual Society for Neuroscience Meeting, New Orleans, 1991.

Correspondence should be addressed to Y. H. Cho, Department of Psychology, University of Utah, Salt Lake City, UT 84112.

Copyright (c) 1993 Society for Neuroscience $0270-6474 / 93 / 131759-08 \$ 05.00 / 0$
}

extend back as far as several years with an identifiable gradient, recent memories being selectively impaired while very remote memories remain intact. These findings provide evidence for a consolidation process that lasts considerably longer than the minutes initially envisioned (for reviews, see Polster et al., 1991; Squire, 1992).

More precisely, this suggests that the creation of permanent memories requires a gradual transformation in the organization of memory traces rather than a simple fixation process. Thus, in line with this idea, it has been proposed that limbic structures remain involved for a lengthy period of time in the organization of enduring extra-limbic memory traces that ultimately become capable of independently supporting the retrieval of information (Squire et al., 1984).

The difficulties inherent to neuropsychological studies aimed at testing memories concerning the remote past, and in addressing the question of how these phenomena are organized in the brain, have recently led to the development of a number of animal models. A series of studies have succeeded in attempts to describe a temporal gradient in retrograde amnesia as evidence for a long-term consolidation process (Squire and Spanis, 1984; Salmon et al., 1987; Sutherland et al., 1987); however, similar neuropsychological studies have failed to find evidence for such a temporal gradient (Dean and Weiskrantz, 1974; Salmon et al., 1987).

The present experiment was mainly aimed at determining whether ibotenate entorhinal cortex (EC) lesions in mice could produce diffcrential and temporally graded retrograde amnesia for successive two-choice discrimination problems, each learned at different time periods prior to surgery. Basically, the method was the same as that previously used by Dean and Weiskrantz (1974) and more recently by Zola-Morgan and Squire (1990). In addition, anterograde amnesia was assessed both by postoperative learning and by rates of forgetting for similar problems as those learned preoperatively.

\section{Materials and Methods}

\section{Subjects}

Twenty-one adult mice of the BALB/C by JIco strain were used. During training, the mice were kept on a food deprivation schedule so that their body weight was maintained at $80-84 \%$ of their free-feeding weight.

\section{Apparatus}

Two identical elevated radial mazes (A and B), located in two different rooms decorated with different sets of pictures and objects, were used. Each maze was composed of a circular platform ( $30 \mathrm{~cm}$ diameter) from which eight arms $(50 \mathrm{~cm}$ long) radiated in a symmetrical fashion. Each arm was fitted with a door at the entrance and a food cup at the other extremity. In maze B, each arm was differentiated by inserts of different 


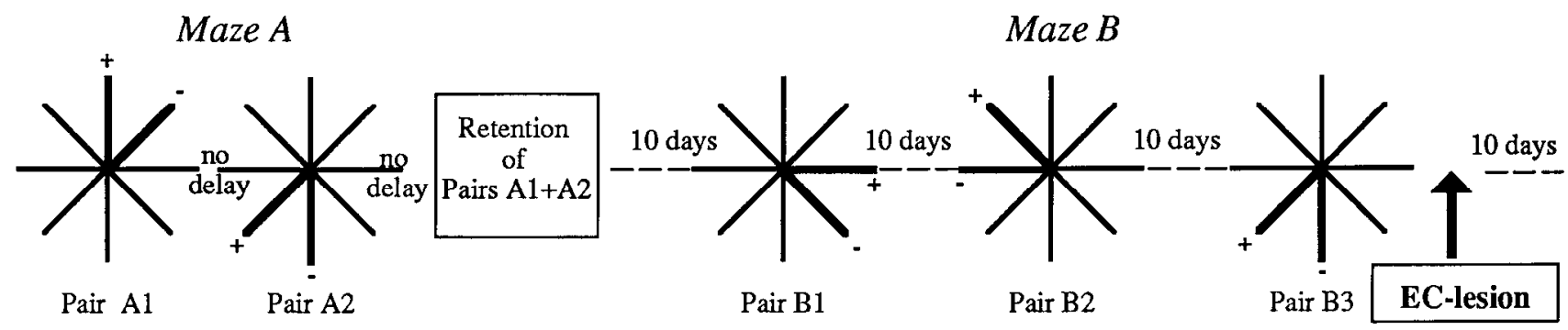

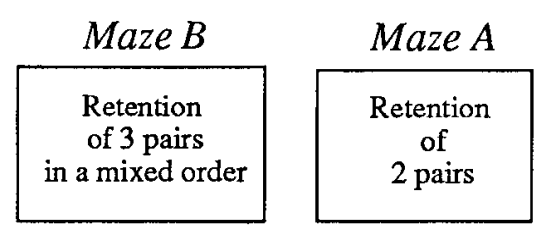

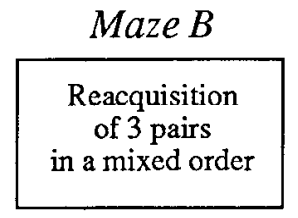

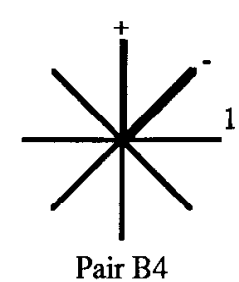

Pair B4

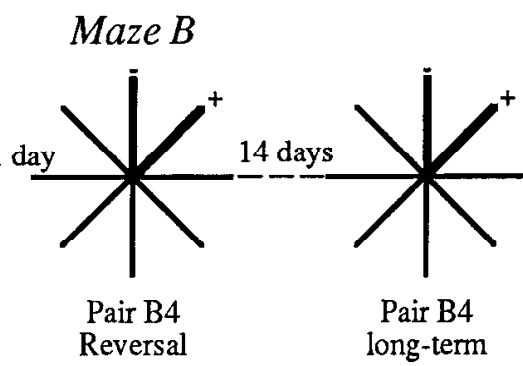

Retention

Figure 1. Behavioral procedure used for studying retrograde and anterograde amnesia following ibotenate EC lesion.

black and white visual cues placed on the floor so that the animals could learn the discrimination tasks on the bases of both extramaze (place) and intramaze cues.

\section{Behavioral testing}

Preoperative training. The gencral proccdure is summarized in Figure 1. All mice were successively trained on five two-choice discrimination problems. For each of the five problems, two adjacent arms were presented, one of which was always baited. Animals were given daily sessions of 16 trials each as follows. At the start of a trial, the mouse was placed on the central platform, and after $15 \mathrm{sec}$, the doors giving access to the first pair of selected arms were opened simultaneously. Once the mouse had reached the food tray (baited or not) at the end of the chosen arm, it was allowed to return to the central platform where it was again confined. After $15 \mathrm{sec}$, the same two doors were opened for the next trial. Training continued (three to five daily sessions) until the animal reached a criterion of 13 correct choices in the same 16-trial session. Training for the second problem (second pair of arms), A2, commenced the next day and was continued until animals attained the same criterion of performance on the second pair of arms. The next set of three discrimination problems was carried out using maze $B$. Thus, at each of five time periods prior to surgery, animals had learned a different twochoice discrimination problem. In maze A only, training for the second problem (A2) began the day after the end of discrimination training for the first pair of arms. Moreover, animals were given an additional session the following day after attaining criterion on pair $A 2$ where both pairs $(\mathrm{A} 1+\mathrm{A} 2)$ were presented in a mixed order. This last session, aimed at familiarizing mice with the mixed-testing procedure used postoperatively in maze B, was given about 6.5 weeks before surgery. Two weeks later ( 4.5 weeks before surgery) animals began the first pair (B1) in maze B; they were subsequently trained on pairs B2 and B3, at around 2.5 weeks and $4 \mathrm{~d}$ before surgery, respectively. The position of the correct arms was counterbalanced across successive pairs, mazes, and subjects. For example, in maze A, the position of the baited arm for pair Al (i.e., right hand) was inverted for pair A2 (i.e., left hand); moreover, the second selected pair of arms (A2) was spatially symmetrical with regard to the first pair (A1). In maze B, the animals were divided in three subgroups. The position of the baited arm (i.e., left or right) was the same for the first and second pairs for first subgroup, and was the same for the first and third pairs, and the second and third pairs for second and third subgroups, respectively, thus precluding the use of simple side-strategies to resolve the problems. Half of each of the three subgroups was attributed to the experimental group and the other half to the sham-operated group.

Postoperative testing. Retention and reacquisition of the preoperatively learned pairs (B1-B3) were assessed in maze B. In each daily session, the three pairs were presented eight times each in a mixed order. Upon completion of testing in maze $\mathrm{B}$, animals were given a single retention session for the two pairs (A1 and A2) learned preoperatively in maze $A$, each presented eight times in a mixed order. Postoperative performance in maze B was assessed on each pair (B1-B3) by the percentage of correct responses recorded on both the first eight trials (first session) and the first 16 trials (first two sessions). Moreover, the number of trials needed to achieve a criterion of five correct responses in five consecutive trials was also determined. The rate of complete reacquisition was assessed by the number of trials needed to reachieve the $13 /$ 16 criterion used for preoperative acquisition. Retention scores in maze $\mathrm{A}(\mathrm{A} 1+\mathrm{A} 2)$ were assessed by the percentage of correct responses recorded on both the first eight and 16 trials of the single retention session.

Postoperative learning, reversal, and retention testing (problem B4). This experiment was designed to evaluate the anterograde effects of EC lesions on learning, reversal, and long-term retention of a new pair. Thus, after completion of postoperative retention tests, animals were trained on the final (fourth) pair in maze B (B4) according to the same procedure as that described for preoperative training. Once the mouse had reached the 13/16 criterion, the baited arm was reversed and training continued until the animal reattained the same criterion. Two weeks later, retention for discrimination reversal was assessed in a single session of 16 trials.

Contribution of intramaze cues to discrimination performance. This last experiment was designed to determine the extent to which subjects used intramaze cues in discrimination. Accordingly, control animals $(n$ $=6)$ and EC-lesioned mice $(n-12)$ were trained concurrently on the four pairs in maze B. During each daily session, the four pairs were presented six times each, in a mixed order, and training continued until animals reached a criterion of 20 correct responses in 24 trials. The following day, they were given a test session during which intramaze visual cues for a given pair were either maintained in the same position (task A), reversed relative to their initial position (task B), or removed (task C). For each of these three tasks, baited arms remained in the same location with respect to extramaze stimuli. For the last task (task D), the cues were reversed (as for task B) but animals were reinforced by choosing the previously baited visual cue. 


\begin{abstract}
Table 1. Preoperative discrimination scores (mean percentage correct) on the last daily session for each of the five pairs learned in both maze $A$ ( $A 1$ and $A 2$ ) and maze $B(B 1, B 2$, and $B 3$ ) in sham- and EC-lesioned groups
\end{abstract}

\begin{tabular}{llllllll} 
& \multicolumn{3}{l}{ Maze A } & & \multicolumn{3}{l}{ Maze B } \\
\cline { 2 - 3 } Group & Pair & Pair & $\begin{array}{l}\text { Pairs } \\
\text { A1 }+\end{array}$ & & Pair & Pair & Pair \\
EC $(n=12)$ & 87.1 & 86.6 & 85.4 & 87.1 & 88.4 & 90.2 \\
Sham $(n=9)$ & 84.4 & 85.2 & 79.2 & 89.1 & 92.2 & 91.4 \\
\hline
\end{tabular}

In maze $A$, animals were given an additional single session with pairs $\mathrm{Al}$ and $\mathrm{A} 2$ presented in a mixed order.

\section{Surgery}

The bilateral lesions of the entorhinal cortex $(n=12)$ were performed using ibotenic acid. Animals were anesthetized with an intraperitoneal injection of pentobarbital $(100 \mathrm{mg} / \mathrm{kg})$ and positioned in a Kopf stereotaxic apparatus. Small quantities of ibotenic acid dissolved in phosphate-buffered saline $(10 \mathrm{mg} / 1 \mathrm{ml})$ were injected using a $1 \mu 1$ Hamilton syringe mounted on the stereotaxic frame. The injections were made at two sites per hemisphere. At the first site, injections were made at 2.0 $\mathrm{mm}(0.03 \mu \mathrm{l}), 3.0 \mathrm{~mm}(0.03 \mu \mathrm{l})$, and $3.5 \mathrm{~mm}(0.04 \mu \mathrm{l})$ ventral to the surface of the brain at AP $-4.3 \mathrm{~mm}$ posterior to bregma, I $+4.0 \mathrm{~mm}$ to midline. At the second site, volumes injected were $\mathrm{V}-1.5 \mathrm{~mm}(0.04$ $\mu \mathrm{l}),-2.0 \mathrm{~mm}(0.04 \mu \mathrm{l})$, and $-3.0 \mathrm{~mm}(0.05 \mu \mathrm{l})$ at AP $-4.8 \mathrm{~mm}, \mathrm{~L}$ $\pm 3.2 \mathrm{~mm}$. Sham-opcrations $(n=9)$ were carried out using the same surgical procedures as for mice in the EC group except for the injection of ibotenic acid. Animals were allowed a recovery period of ten days before the start of postoperative retention tests.

\section{Histology}

On completion of the experiment all animals were perfused intracardially with physiological saline and formalin. The brains were removed, fixed in formalin-sucrose, and sectioned horizontally on a microtome at $40 \mu \mathrm{m}$. The brain sections were stained using thionin for histological examination of the position and extent of the lesions.

\section{Results}

Histology

Examination of the brain sections of all experimental subjects $(n=12)$ revealed extensive bilateral damage to the entorhinal cortex extending fully along the dorsoventral area. Eight EClesioned mice revealed somewhat limited and selective bilateral damage to the entorhinal cortex extending along the dorsoventral area (Fig. 2, top right). The damage included both lateral and medial subdivisions of the entorhinal cortex, and occasionally some cells of the ventral subiculum. At the most ventral level, there was an inconsistent loss of cells. The other four experimental mice showed, in addition to the extensive EC lesion, some loss of cells in the caudal dentate gyrus, ventral subiculum, and especially adjacent ventral CA1-CA3 subfields of the right hippocampus across the dorsoventral level (Fig. 2, bottom left).

In all cases, damage to the lateral part of the entorhinal cortex was more pronounced than that to the medial part.

The nine animals in the sham-operated group exhibited no apparent damage except for a slight increase in glial cells at the site where the micropipette penetrated the neocortex.

\section{Behavior}

\section{Preoperative training}

Animals learned the first pair (maze A, 6.5 weeks prior to surgery) in an average of 4.35 sessions ( 16 trials per session). The

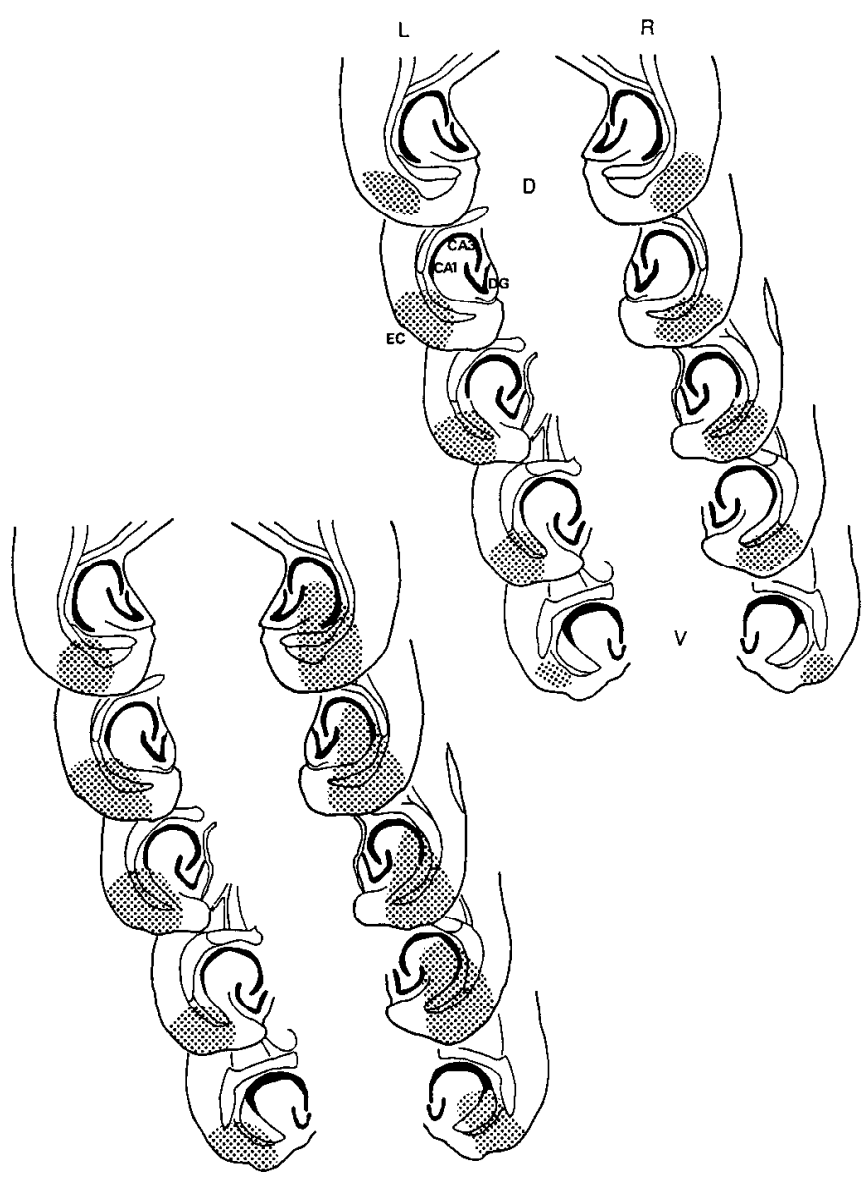

Figure 2. Horizontal reconstructions [from dorsal $(D)$ to ventral $(V)$ ] of lesion placement and size of each representative subject of the case of the lesion limited to the entorhinal cortex including both medial and lateral part (top right) and the case of EC lesion combined to that of adjacent right ventral CA1-CA3 ficlds of the hippocampus (bottom left). $E C$, entorhinal cortex; $C A 1$ and $C A 3$, fields of the hippocampus; $D G$, dentate gyrus; $L$, left; $R$, right.

subsequent four pairs (A2, B1, B2, and B3) were learned more quickly [respectively, $2.18,2.54,2.36$, and 2.46 sessions; trend analysis on the five pairs: $F(1,19)=69.0, p<0.001$ ]. Successive learning curves and sessions to criterion for the five pairs were comparable for both animals that underwent EC lesioning and for sham-operated subjects $(F$ values $<1)$. Finally, as shown in Table 1, discrimination scores on the last day of training for each of the three pairs learned in maze B (B1-B3) did not differ between pairs and groups $(F$ values $<1)$. Although animals of the sham-lesioned group were slightly inferior to the EC-lesioned mice when tested on pairs A1 and A2 (79.2\% vs. $85.4 \%)$, this was not statistically significant $[F(1,19)=2.66 ; p>0.10]$.

\section{Postoperative retention tests}

Results are summarized in Figure 3 and Table 2.

Maze B. Whatever the measure used, sham-operated mice ( $n$ =9) exhibited a significant forgetting of the three discrimination problems across the 5.5 week time period [first eight trials (day 1): from $84.7 \%$ for the third pair learned $10 \mathrm{~d}$ earlier (B3) to $62.5 \%$ for the first one learned 5.5 weeks before testing (B1); trend analysis, three pairs: $F(2,16)=13.5, p<0.001$; first 16 trials: from $87.5 \%$ for the third pair (B3) to $66.7 \%$ for the first one (B1) $(p<0.001)$, and from 6.2 trials (B3) to 18.0 trials (B1) 


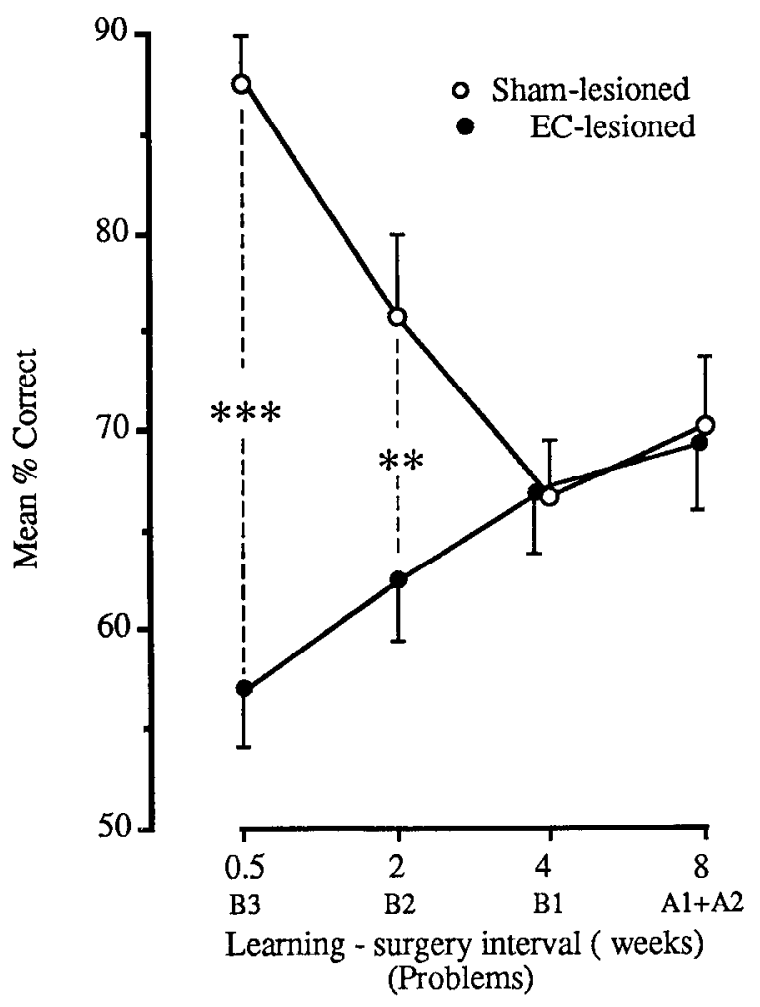

Figure 3. Postoperative retention scores on the first 16 trials (mean \pm SEM) in EC-lesioned $(O)$ and sham-lesioned $(O)$ animals as a function of the learning-surgery interval. Retention of pairs B1, B2, and B3 learned in maze $B$ was assessed $10 \mathrm{~d}$ after surgery by presenting each of the three pairs in a mixed order. Retention of the two pairs (A1 + A2) learned in maze $A$ was assessed separately $12 \mathrm{~d}$ after surgery. ***, $p<0.001$ in comparison to the sham-lesioned group; ${ }^{* *}, p<0.01$.

to reach the $5 / 5$ criterion $(p<0.05)$ ]. Finally, it must be noted that discrimination scores recorded on the second day of testing were slightly, but not significantly, better than those recorded on the first day [pooled three pairs, 24 trials, day 2 vs. day 1: $79.6 \%$ vs. $73.6 \% ; F(1,8)=3.4 ; p=0.10$ ].

In contrast, performances of EC-lesioned mice $(n=12)$ tended to improve as the retention interval increased. Thus, experimental subjects exhibited better performance for remote information (first learned pair, B1) than for recently acquired information (third pair, B3) both on the first 16 trials $[67.2 \%$ vs. $56.8 \% ; F(1,11)=6.0 ; p=0.03$ ] and on the number of trials needed to achieve the $5 / 5$ criterion ( 13.0 vs. 38.8 trials; Wilcoxon signed-rank test, $z=-2.19 ; p<0.02)$. However, even though the mean discrimination scores recorded on the first 8 trials was significantly above chance level $(50 \%)$ on the first (B1, $67.7 \% ; p<0.01$ ) but not on the third pair (B3, 56.3\%; $p>$ 0.30 ), the difference between these two pairs did not reach statistical significance $[F(1,11)=2.4 ; p=0.15]$. Finally, there were no significant differences between discriminations scores observed on the first and second days of testing, both on the pooled three pairs ( 24 trials, day 1 vs. day 2 : from $61.5 \pm 2.9 \%$ to 62.2 $\pm 1.8 \%$ ) and on each pair (eight-trial blocks, day 1 vs. day 2 : $F$ values $<0.12$ for all comparisons). Moreover, these slight changes were not significantly different from those observed in sham-lesioned mice [group $\times$ day interactions for the pooled three pairs: $F(1,19)=0.94$; for each pair: $F(1,19)<1.6 ; p\rangle$ $0.20]$.

Maze A. Experimental and control groups exhibited above chance $(p<0.01)$ and comparable discrimination scores both on the first eight $(67.7 \%$ and $73.6 \%$, respectively) and first 16 trials $(69.3 \%$ and $70.1 \%)$ of the single session for the two pairs $(\mathrm{A} 1+\mathrm{A} 2)$ learned 7 weeks earlier.

\section{Reacquisition in maze $B$}

Results are summarized in Table 2. For sham-lesioned animals, the mean number of trials needed to reachieve the 13/16 acquisition criterion on each of the three pairs (B1-B3) tested concurrently (eight trials per pair in each session) decreased as the retention interval increased [trend analysis: $F(2,16)=7.0$, $p<0.01]$. In contrast, an inverse but nonsignificant trend was observed in EC-lesioned subjects [trend analysis: $F(2,22)=1.73$, $p=0.20]$; lesioned animals performed significantly worse than controls [group: $F(1,19)=25.4, p<0.001$ ] with a significant group $\times$ pair interaction $[F(2,38)=5.6 ; p<0.01]$.

\section{Postoperative learning, reversal, and retention testing (fourth pair in maze B)}

As shown in Figure 4A, EC-lesioned animals learned the novel fourth pair (B4) in maze B at the same rate as controls [first 3 d; $F(2,38)=0.69$ ] and reached the criterion in a slightly, but not significantly, higher number of sessions $[4.1$ vs. $2.9 ; F(1,19)$ $=1.46 ; p>0.20]$. In contrast, reversal learning (Fig. $4 B$ ) was significantly impaired in EC-lesioned as compared to shamlesioned animals across the three first sessions $[F(1,19)=7.5$; $p<0.02$ ], though the difference in the number of sessions to criterion did not reach significance $[4.8$ vs. $3.3 ; F(1,19)=3.6$;

Table 2. Mean percentage correct responses ( \pm SEM) on the first eight trials (day 1) of retention in maze $A$ and maze $B$, and mean number of trials ( $\perp$ SEM) needed to achieve the $5 / 5$ and $13 / 16$ criteria (maze $B$ only) on each pair learned at different intervals (in parentheses) before surgery in sham- and EC-lesioned animals

\begin{tabular}{|c|c|c|c|c|c|c|}
\hline Measure & Group & B3 ( 0.5 week) & B2 (2 weeks) & B1 (4 weeks) & $\begin{array}{l}\text { Trend analysis } \\
\text { Maze B }\end{array}$ & $\begin{array}{l}\mathrm{A} 1+\mathrm{A} 2 \\
(8 \text { weeks })\end{array}$ \\
\hline $\begin{array}{l}\% \text { correct, } \\
\text { first } 8 \text { trials }\end{array}$ & $\begin{array}{l}\text { Sham } \\
\text { EC }\end{array}$ & $\begin{array}{l}84.7 \pm 2.8 \\
56.3 \pm 4.7^{* * *}\end{array}$ & $\begin{array}{l}73.6 \pm 4.4 \\
60.4 \pm 4.6\end{array}$ & $\begin{array}{l}62.5 \pm 4.2 \\
67.7 \pm 4.7\end{array}$ & $\begin{array}{l}p<0.001 \\
\text { NS }\end{array}$ & $\begin{array}{l}73.6 \pm 4.4 \\
67.7 \pm 5.6\end{array}$ \\
\hline $\begin{array}{r}5 / 5 \text { criterion } \\
\text { (no. trials) }\end{array}$ & $\begin{array}{l}\text { Sham } \\
\text { EC }\end{array}$ & $\begin{array}{l}6.2 \pm 0.5 \\
38.8 \pm 10.1^{* * *}\end{array}$ & $\begin{array}{l}11.9 \pm 3.4 \\
27.2 \pm 7.6\end{array}$ & $\begin{array}{l}18.0 \pm 4.4 \\
13.0 \pm 2.0\end{array}$ & $\begin{array}{l}p=0.03 \\
p=0.02\end{array}$ & $\begin{array}{l}- \\
-\end{array}$ \\
\hline $\begin{array}{c}13 / 16 \text { criterion } \\
\text { (no. trials) }\end{array}$ & $\begin{array}{l}\text { Sham } \\
\text { EC }\end{array}$ & $\begin{array}{l}20.4 \pm 2.4 \\
47.5 \pm 4.9^{* * *}\end{array}$ & $\begin{array}{l}25.8 \pm 4.0 \\
49.8 \pm 4.6^{* *}\end{array}$ & $\begin{array}{l}35.6 \pm 2.7 \\
40.0 \pm 3.3\end{array}$ & $\begin{array}{l}p<0.01 \\
\text { NS }\end{array}$ & - \\
\hline
\end{tabular}




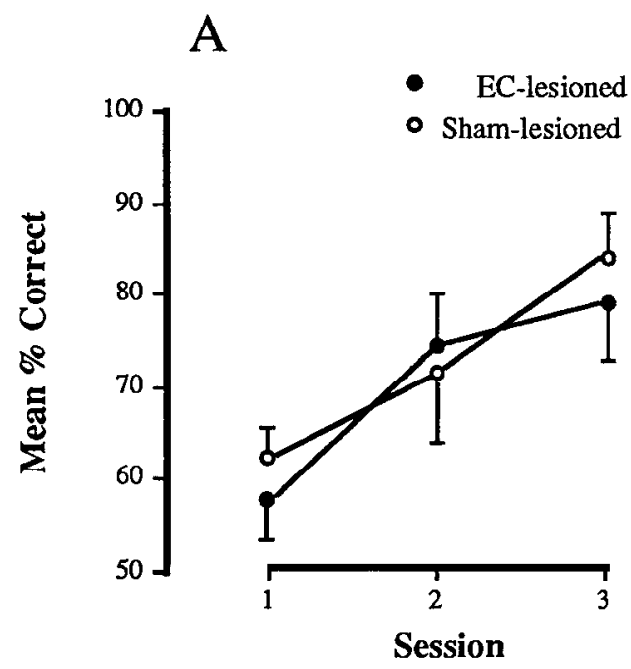

B

$\mathrm{C}$
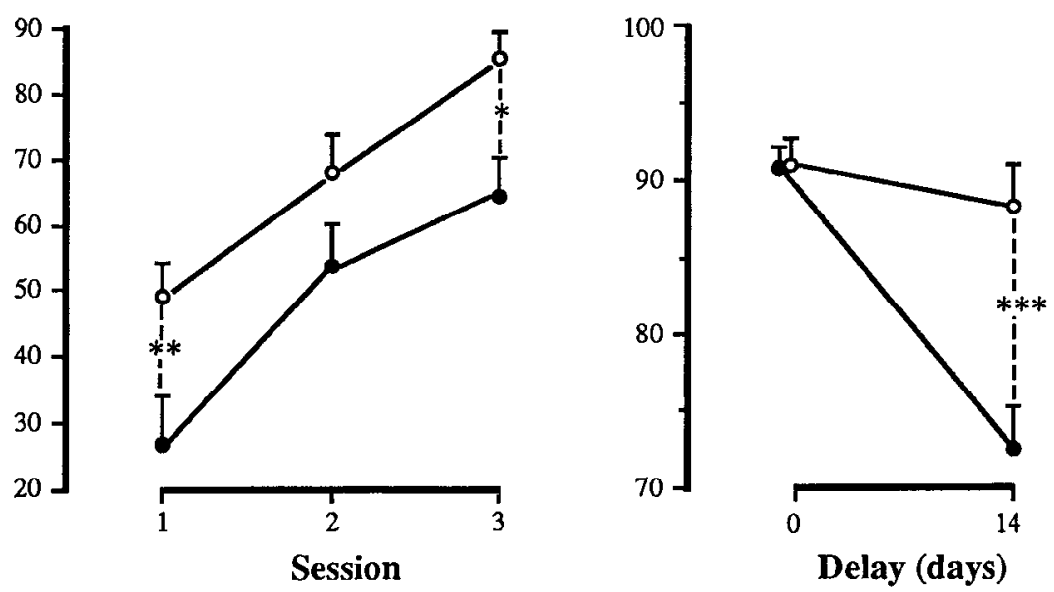

Figure 4. Discrimination performances of EC-lesioned (O) and sham-lesioned (O) animals tested on the fourth pair in maze B (B4): acquisition $(A)$ and reversal $(B)$ learning curves across the three first daily sessions of each stage. $C$, Discrimination performance on the last reversal learning session (delay 0 ) and on the 2 week delayed retention session. ${ }^{*}, p<0.05 ;{ }^{* *}, p<0.01 ;{ }^{* * *}, p<0.001$; in comparison to the sham-lesioned group.

$p=0.07]$. In fact, four EC-lesioned mice were still responding at levels below chance $(50 \%)$ on the third session and two of them were unable to achieve the criterion within seven sessions. These two subjects were discarded for subsequent testing. As shown in Figure $4 C$, EC-lesioned animals that exhibited the same level of performance as controls on the last reversal training session (90.6\% vs. $91.0 \%$ ) displayed a faster rate of forgetting over the two weeks retention interval [groups $\times$ delays interaction: $F(1,17)=13.8, p<0.01]$.

\section{Relationships between postsurgery learning deficits and retrograde amnesia}

As compared to all other animals (sham- and EC-lesioned), four EC-lesioned subjects exhibiting unilateral lesions of the hippocampal CA subfields (Fig. 2, bottom left), were disproportionally impaired on the acquisition and reversal of the pair B4 (see previous section). However, as can be seen in Table 3, these subjects were indistinguishable from the others in their retention scores on the pair B1 learned four weeks before surgery, and from the other eight EC-lesioned animals for retention scores on the most recently acquired pairs (B2 and B3).

\section{Contribution of intramaze cues to discrimination performance}

Results are summarized in Figure 5. Changing the location (task B) or removing intramaze cues (task C) produced an overall decrease in discrimination performance on the three tasks (including that of $\mathrm{A}$, cues unchanged) for which baited arms remained in the same location with respect to extramaze stimuli [three tasks; $F(2,28)=5.79 ; p<0.01]$. However, more importantly, performances recorded on the test session were not significantly different among groups $[F(1,14)=1.86 ; p>0.10]$ and tasks $[F(2,28)=0.73, \mathrm{NS}]$ with no significant group $\times$ task interaction $[F(2,20)=0.92$, NS]. For task $\mathrm{D}$, in which the location of the baited and nonbaited cues were reversed with respect to extramaze stimuli, discrimination performance dropped below chance level in both groups [31.3\% and $41.3 \%$ for, respectively, EC- and sham-lesioned groups; $F(1,14)=1.91$, NS].

\section{Discussion}

The main finding of this experiment is that bilateral ibotenate lesions of the entorhinal cortex produce a lengthy and temporally graded retrograde amnesia (about 4.5 weeks). Thus, when tested concurrently on three two-choice discrimination problems, each learned at different time periods (maze B), control mice exhibited significant forgetting with increasing retention intervals (from about 2 weeks to 6 weeks). In contrast, operated animals actually exhibited better retention scores for the less recently learned pair (i.e., 4.5 weeks before surgery) than for the two pairs learned within 2 weeks prior to surgery. Finally, for

Table 3. Mean number of sessions ( \pm SEM) necessary to acquire pair B4 (Acquisition) and its reversal (Reversal) in maze B, and total of two acquisitions $(A+R)$ in sham, and two EC groups $(n=4$ and 8$)$ subdivided by their different patterns of new learning scores

\begin{tabular}{|c|c|c|c|c|c|}
\hline \multirow[b]{2}{*}{ Group } & \multicolumn{3}{|c|}{ Pair B4 (postsurgery) } & \multirow{2}{*}{$\begin{array}{l}\text { Pair B1 } \\
\text { reacquisition (\%) }\end{array}$} & \multirow{2}{*}{$\begin{array}{l}\text { Pair } \mathrm{B} 2+\mathrm{B} 3 \\
\text { reacquisition (\%) }\end{array}$} \\
\hline & Acquisition & Reversal & $A+R$ & & \\
\hline \multicolumn{6}{|l|}{$\mathrm{EC}$} \\
\hline$(n=4)$ & $7.3 \pm 1.3$ & $7.5 \pm 0.6$ & $14.8 \pm 1.3$ & $4.3 \pm 0.7(70.4 \pm 8.2)$ & $6.3 \pm 1.1(59.4 \pm 2.2)$ \\
\hline \multicolumn{6}{|l|}{ Sham } \\
\hline$(n=9)$ & $2.9 \pm 0.4$ & $3.3 \pm 0.4$ & $6.2 \pm 0.4$ & $4.4 \pm 0.3(66.7 \pm 2.8)$ & $2.9 \pm 0.3(81.7 \pm 2.9)$ \\
\hline
\end{tabular}




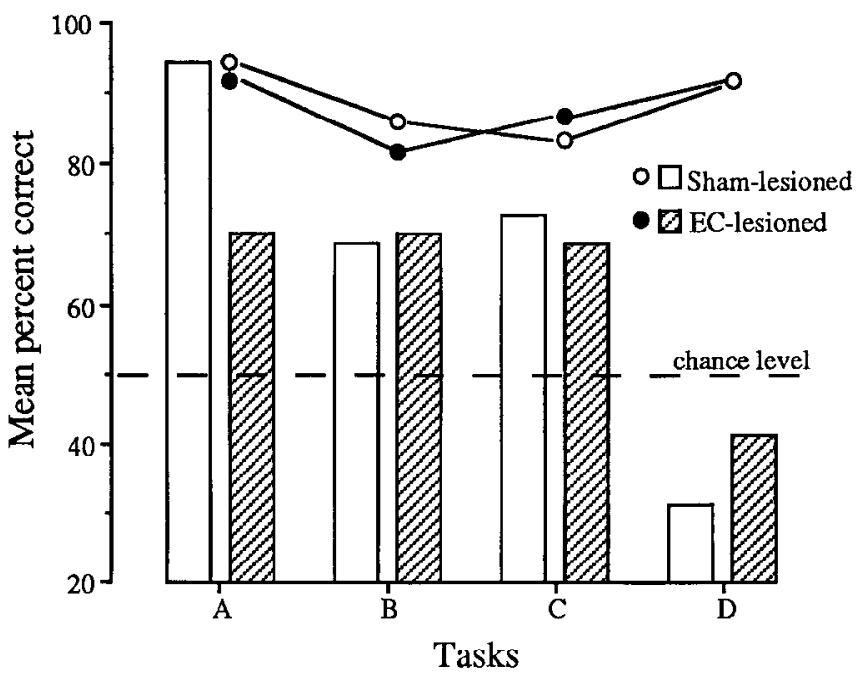

Figure 5. Mean correct percentages on the last session of reacquisition of four tasks with initial cue positions $(O$, sham-operated controls; $\bullet$, EC-lesioned animals), and those recorded in modified cue positions for each of four tasks (open bars, sham-operated controls; shaded bars, EClesioned animals). For that, intramaze visual cues were either maintained in the same position (task $A$ ), reversed relative to their initial one (tasks $B, D$ ) or removed (task $C$ ). The baited arms remained in the same spatial location for all tasks except for the task $D$, for which the baiting arm is matched with visual cue.

both the pairs in maze B (B1) and the two pairs in maze A (A1 and $A 2$ ) learned about 4.5 and 6.5 weeks before surgery, EClesioned animals exhibited the same retention scores as shamoperated controls. Taken as a whole, these results indicate that a 4 week period is required before memory for a two-choice discrimination problem no longer depends on the integrity of the entorhinal cortex; they also suggest that, beyond this time, an EC-independent memory storage system is capable of supporting the retrieval of information.

A long temporal gradient for retrograde amnesia ranging from $2 \mathrm{~d}$ to 12 weeks has been previously reported in animals following electroconvulsive shock (Squire and Spanis, 1984) and hippocampal lesions (Sutherland et al., 1987; Winocur, 1990; Zola-Morgan and Squire, 1990). These findings suggest that, even though different tasks, species, and treatments might generate different estimates of the length of the consolidation period, there exists a general consensus for a consolidation process that lasts considerably longer than the seconds or minutes initially envisioned (see Squire, 1992, for a review).

It must be mentioned, however, that in our present cxperiment none of the measurements of retention that were used may be considered as a pure probe of memory since more than a single (the first) trial was necessary to assess discrimination performance reliably on each pair. Indeed, whereas on the first trial, the animal's choice may be considered as entirely dependent on the retrieval of information learned before surgery, choices subsequent to the first trial may additionally benefit from information obtained by the outcome of the preceding trials. Thus, even though, in both groups, we did not observe significant relearning between the first and second day of retention testing, one cannot totally rule out a contamination of retention measurements by relearning processes that would have occurred at the very beginning of the first retention session. A comparison of the number of trials needed to achieve successively the $5 / 5$ and $13 / 16$ criterions in the experimental group indicated that, whereas animals required significantly less trials to reach the $5 / 5$ criterion for remote (B3) than for recently acquired information (B1), this difference was reduced and no longer significant for the subsequent $13 / 16$ criterion (see Table 2 ). An opposite pattern of results has in fact been reported by Sutherland et al. (1987), who showed that retrograde amnesia produced in rats by hippocampal damage was more pronounced at the end than at the beginning of retention, in a test of spatial reference memory in the Morris water maze task. One possible explanation for this discrepancy (among others) could be the degree of anterograde learning impairment produced by the lesion with respect to the task considered. It seems, however, that as a general rule, there is a dissociation between the effects of lesions of the hippocampal formation on acquisition behavior and retention. Thus, retrograde amnesia has been reported on tasks for which learning was either impaired (Sutherland et al., 1987), unimpaired (Winocur, 1990), or even facilitated (Sara, 1981). These observations could be of theoretical importance with respect to the dissociation between the role of the hippocampus as a (temporary) neuronal substrate for memory traces and its further involvement in memory consolidation (see below).

Coming back to our own results, it seems quite clear that ibotenate lesions of the entorhinal cortex produced little, if any, impairment for the acquisition of the new (B4) pair; moreover, even for the four subjects that were actually impaired on postoperative learning (probably because of extra-entorhinal damage), there was no evidence at all for a greater impairment of remote memory than in other animals (see Table 3). Together with the observation that, over the 2 week period following postoperative acquisition, lesioned mice showed faster forgetting than controls (antcrogradc amncsia), this suggcsts that both the anterograde and retrograde amnesia observed are more likely to reflect a loss of memory rather than an impairment of reacquisition.

It is now important to consider the type of memory that was affected by EC lesions in this study. Before starting the experiment we speculated that successive discriminations would be better retained over long periods if animals were provided with intramaze in addition to extramaze distal cues. In fact, our results suggest that, at least in overtrained animals, the contribution of intramaze cues to discrimination scores was quite low. First, data from preoperative learning showed that shifting from the last noncued task of maze A (A2) to the first cued one in maze $B$ (B1) had no detectable facilitative effect on the speed of acquisition; similarly, no differences were observed between postoperative retention scores for the cued (B1) and the two noncucd (A1 I A2) tasks, which, morcover, werc both tested at a longer retention interval than the first one (see Fig. 3). Second, the fact that animals continued to discriminate correctly and significantly between arms after intramaze cues had been either reversed or removed for a given task, strongly suggests that they mainly used distal cues. Indeed, even though mice from both groups were disturbed by these changes, as shown by their overall decrease in performance, no significant differences were observed between tasks for which a change in intramaze cues was carried out and the one for which it was not (see Fig. 5). Thus, it seems reasonable to postulate that animals solved the task by using relationships between distal elements of the environment (i.e., spatial mapping; O'Keefe and Nadel, 1978) or other configural associations (Sutherland and Rudy, 1989) rather than simple associations. In these conditions, the hippocampal formation, in concert with posterior cortical areas, 
would initially be involved in the internal representation of this type of information.

Since, after a sufficient amount of time (about 4 weeks) the entorhinal cortex seems no longer to be necessary to sustain normal remembering, one might suppose that normal behavioral forgetting actually reflects "hippocampal forgetting." This would suggest a close relationship not only between the rates of behavioral forgetting for spatial information and decay of hippocampal long-term synaptic enhancement (Barnes and McNaughton, 1985), but also between these two phenomena and the temporal gradient of retrograde amnesia. Whatever be the case, there is no reason to believe that the internal representation that survives hippocampal forgetting or lesioning holds exactly the same information (i.e., a duplication, even though weakened) as that held earlier by, presumably, both hippocampal and cortical substrates. Assumptions concerning a dynamic and evolutive process of memory representations in the brain have been suggested on several occasions, for example, "engram migration" during interhemispheric transfer of monocularly acquired visual discrimination (Bures and Buresova, 1990), "memory spread" over time from the hippocampal-entorhinal area to widespread forebrain areas (Flexner et al., 1991), and "flow of memory" generated by a one-trial passive avoidance task from intermediate medial hyperstriatum ventrale to lobus parolfactorius in chicks (Rose, 1991).

In the context of the present experiment, this means that, as time passes, some subtle changes may occur in the strategy the animal uses on the retention test, as long as this strategy has a sufficiently adaptive value with respect to the demands of the task. We have begun to investigate such a possibility. First, even though subjects tested for rcmote memory (4 weeks or more following learning) exhibited performances well above chance levels, they generally behaved as if they did not remember the discrimination problems (i.e., they did not hesitate before choosing as they did for recent memories); although this may represent a rather anthropomorphic argument, it would suggest that animals solved the task on the basis of less but sufficient information than initially. Second, recent experiments have provided indirect evidence that the tendency to use an egocentric strategy becomes more prominent as the retention interval increases. More precisely proactive interference produced by a previously learned task on the acquisition of the following one, for which position of the correct arm relative to the animals' position was reversed (i.e., right hand then left hand or inversely), was more pronounced for long ( 3 weeks) than for short ( $1 \mathrm{~d})$ intervals ( $Y$. H. Cho and R. Jaffard, unpublished observations). Finally, the fact that some units in the rat parictal cortex have been found to fire during particular movements (i.e., right or left turns) in conjunction with local features (i.e., visual stimuli) (Leonard and McNaughton, 1990; McNaughton et al., 1990) would agree with the idea that only this kind of subset of the whole initial representation would ultimately be maintained in the posterior parietal cortex. In this sense, the formation of remote memory for the type of events we have sludied would involve a "translocation" of the internal representation and a change in its code. The fact that, for these events, retrograde amnesia was temporally graded, also suggests that during a period of about 4 weeks the entorhinal cortex remains necessary to organize (or consolidate) the cortical memory trace fully.

At this point, it seems necessary to return again to the lack of effect of $\mathrm{EC}$ lesion on postoperative acquisition of the B4 task. Indeed, this finding, together with some other ones (see above) seems to contradict the hypothesis that, initially, the hippocampal formation supports a subset of the whole information. In the task we have used, however, EC-lesioned animals that were given no or less important preoperative training than the subjects of the present experiment were actually impaired (Cho and Jaffard, unpublished observations). As pointed out by several authors, this emphasizes the importance of preoperative training in deciding whether a given lesion impairs a given task (Barnes, 1988; Jarrard, 1991). This suggests that the "learning to learn" phenomenon, as well as learning a simple two-choice discrimination problem, may be spared by EC lesions, provided animals are given a minimum degree of preoperative training, with a sufficiently long delay before the lesion is performed. Therefore, this would mean that, as preoperative training progressed, the need for a hippocampal representation of events that must be remembered in the same context and requiring the same basic rule (learning set) diminishes.

Since, despite this spared learning ability, lesioned animals nevertheless exhibited anterograde amnesia, this could reflect a dissociation between the two classically hypothesized functions of the hippocampal formation, that is, as a temporary storage site for certain kinds of information (Rawlins, 1985) and as a consolidation-organizer device (Squire, 1992). More precisely, unless one considers that consolidation is a simple fixation process, it is difficult to imagine how the hippocampal formation could influence and organize extra-hippocampal memory traces without storing any index of the original experience (cf. Teyler and DiScenna, 1986).

\section{References}

Barnes CA (1988) Spatial learning and memory process: the search for their neurobiological mechanisms in the rat. Trends Neurosci 11: 163-169.

Barnes CA, McNaughton BL (1985) An age comparison of the rates of acquisition and forgetting of hippocampal synapses. Behav Neurosci 99:1040-1048.

Bures J, Buresova O (1990) Reversible lesions allow reinterpretation of system level studies of brain mechanisms of behavior. Concepts Neurosci 1:69-89.

Dean P, Weiskrantz L (1974) Loss of preoperative habits in rhesus monkeys with inferotemporal lesions: recognition failure or relearning deficit? Neuropsychologia 12:299-311.

Flexner LB, Flexner JB, Church AC (1991) Long-term suppression in mice of the development of complementary memory storage sites: effect of a muscarinic antagonist. Pharmacol Biochem Behav 39:689694.

Jarrard LE (1991) On the neural bases of the spatial mapping system: hippocampus vs. hippocampal formation. Hippocampus 1:236-239.

Leonard B, McNaughton BL (1990) Spatial representation in the rat: conceptual, behavioral and neurophysiological perspectives. In: The neurobiology of comparative cognition (Kesner R, Olton DS, eds), pp 363-422. Hillsdale, NJ: Erlbaum.

McNaughton BL, Leonard B, Chen L (1990) Cortico-hippocampal interactions and cognitive mapping: $a$ hypothesis based on reintegration of the parietal and inferotemporal pathways for visual processing. In: Learning and memory (Squire LR, Mishkin M, Shimamura A, eds), pp 89-101. New York: Elsevier.

O'Keefe J, Nadel L (1978) The hippocampus as a cognitive map. Oxford: Clarendon.

Polster AR, Nadel L, Schacter DL (1991) Cognitive neuroscience analyses of memory: a historicial perspective. J Cogn Neurosci 3:95-116.

Rawlins JNP (1985) Associations across time: the hippocampus as a temporary memory store. Behav Brain Sci 8:479-528.

Kose SPR (1991) How chicks make memories: the cellular cascade from c-fos to dendritic remodelling. Trends Neurosci 14:690-697.

Salmon DP, Zola-Morgan S, Squire LR (1987) Retrograde amnesia following combined hippocampal-amygdal lesions in monkeys. Psychobiology 15:37-47. 
Sara SJ (1981) Memory deficits in rats with hippocampal or cortical lesions: retrograde effect. Behav Neural Biol 32:504-509.

Squire LR (1992) Memory and the hippocampus: a synthesis from findings with rats, monkeys, and humans. Psychol Rev 99:195-231.

Squire LR, Spanis CW (1984) Long gradient of retrograde amnesia in mice: continuity with the findings in humans. Behav Neurosci 98 : 345-348.

Squire LR, Cohen NJ, Nadel L (1984) The medial temporal region and memory consolidation: a new hypothesis. In: Memory consolidation (Weingartner $\mathrm{H}$, Parker E, eds), pp 185-210. Hillsdale, NJ: Erlbaum.

Sutherland R, Rudy JW (1989) Configural association theory: the role of the hippocampal formation in learning, memory, and amnesia. Psychobiology 17:129-144.
Sutherland R, Arnold KA, Rodriguez AR (1987) Anterograde and retrograde effects on place memory after limbic or diencephalic damage. Soc Neurosci Abstr 13:1066.

Teyler TJ, DiScenna P (1986) The hippocampal memory indexing theory. Behav Neurosci 100:147-154.

Winocur G (1990) Anterograde and retrograde amnesia in rats with dorsal hippocampal or dorsomedial thalamic lesions. Behav Brain Res 38:145-154.

Zola-Morgan SM, Squire LR (1990) The primate hippocampal formation: evidence for a time-limited role in memory storage. Science 250:288-290. 